\title{
LINEAR DETERMINING EQUATIONS FOR DIFFERENTIAL CONSTRAINTS
}

\author{
OLEG V. KAPTSOV and ALEXEY V. SCHMIDT \\ Institute of Computing Modelling RAS, Akademgorodok, 660036, Krasnoyarsk, Russia \\ e-mail:kaptsov@ksc.krasn.ru
}

(Received 10 November, 2003; accepted 1 October, 2004)

\begin{abstract}
A construction of differential constraints compatible with the GibbonsTsarev equation is considered. Certain linear determining equations with parameters are used to find such differential constraints. They generalize the classical determining equations that are used in searching for Lie operators. We introduce the notion of an invariant solution under an involutive distribution and give sufficient conditions for existence of such solutions.
\end{abstract}

2000 Mathematics Subject Classification. 34G20, 35K57.

1. Introduction. As is well known, one can produce many exact solutions of partial differential equations by means of additional constraints [1], [2]. Differential constraints arose originally in the theory of partial differential equations of the first order. Lagrange in particular used differential constraints to find the total integral of the nonlinear equation

$$
F\left(x, y, u, u_{x}, u_{y}\right)=0 .
$$

Darboux [3] applied differential constraints to integrate partial differential equations of second order. The detailed description of the Darboux method can be found in $[1],[4]$.

The general formulation of the method of differential constraints requires that the original system of partial differential equations

$$
F^{1}=0, \ldots, \quad F^{m}=0
$$

be enlarged by appending additional differential equations (differential constraints)

$$
h_{1}=0, \ldots, \quad h_{p}=0,
$$

such that the over-determined system (1.1), (1.2) satisfies some conditions of compatibility.

The theory of over-determined systems was developed by Delassus, Riquier, Cartan, Ritt, Kuranishi, Spencer and others. One can find references in the book of Pommaret [5]. Now the applications of over-determined systems include such diverse fields as differential geometry, continuum mechanics and nonlinear optics. Unfortunately the problem of finding all differential constraints compatible with certain equations can be more complicated than the investigation of the original 
equations. Therefore it is better to content oneself with finding constraints in some classes, and these classes must be chosen using additional considerations.

Recently a new method was proposed for finding differential constraints, which uses linear determining equations. These equations are more general than the classical determining equations for Lie generators [6] and depend on some parameters. Given an evolution equation

$$
u_{t}=F\left(t, x, u, u_{1}, \ldots, u_{n}\right)
$$

where $u_{k}=\frac{\partial^{k} u}{\partial x^{k}}$, then according to [7] the linear determining equation corresponding to (1.3) is of the form

$$
D_{t}(h)=\sum_{i=0}^{n} \sum_{k=0}^{i} b_{i k} D_{x}^{i-k}\left(F_{u_{n-k}}\right) D_{x}^{n-i}(h), \quad b_{i k} \in R .
$$

Here, and throughout, $D_{t}, D_{x}$ are the operators of total differentiation with respect to $t$ and $x$. Equality (1.4) must hold for all solutions of (1.3). The function $h$ may depends on $t, x, u, u_{1}, \ldots, u_{p}$. The number $p$ is called the order of the solution of the equation (1.4). If we have some solution $h$, the corresponding differential constraint is

$$
h=0 \text {. }
$$

It was also shown in [7] that the equations (1.4) and (1.5) constitute a compatible system. Applications of this approach to diffusion equations can be found in [8].

The organization of this chapter is as follows. In section 2 we focus on solutions of second and third order to the linear determining equation for the Gibbons-Tsarev equation [9]

$$
u_{t t}=u_{x} u_{t x}-u_{t} u_{x x}+1
$$

This gives the corresponding differential constraints and allow us to find some exact solutions of (1.6). In the final section, the invariant solutions under an involutive distribution are discussed. We consider the problem of finding involutive distributions that enable us to obtain invariant solutions to evolution equations.

2. Gibbons-Tsarev equation. In this section we will consider the Gibbons-Tsarev equation [9]

$$
z_{x x}+z_{y} z_{x y}-z_{x} z_{y y}+1=0,
$$

which arises in reductions of the Benney equation.

The linear determining equation has the form

$$
D_{x}^{2} h+z_{y} D_{x} D_{y} h-z_{x} D_{y}^{2} h+b_{1} z_{y y} D_{x} h+b_{2} z_{x y} D_{y} h=0,
$$

the constants $b_{1}$ and $b_{2}$ are to be determined together with the function $h$. It can be shown that the equation (2.2) has a solution of the form

$$
h=z_{y y}+g\left(x, y, z, z_{x}, z_{y}, z_{x x}\right)
$$


if and only if the function $g$ is independent of $z_{x x}$. Therefore we shall start with solutions of the second order

$$
h=z_{y y}+g\left(x, y, z, z_{x}, z_{y}\right) .
$$

Substituting (2.3) into (2.2) leads to an equation which includes derivatives of the third order. We can express all mixed derivatives by means of (2.1). Setting the coefficients of $z_{x x x}$ and $z_{y y y}$ equal to zero we obtain:

$$
b_{1}=1, \quad b_{2}=-1 .
$$

The left-hand side of (2.2) is a polynomial with respect to $z_{x x}$ and $z_{y y}$. This polynomial must identically vanish. Collecting similar terms we have the following equations:

$$
\begin{aligned}
& p g_{p p}+q g_{p q}-g_{q q}+2 g_{p}=0 \\
& \quad\left(-q^{2}-2 p\right) g_{p p}+2 g_{q q}+q^{2} g_{x p}+q\left(q^{2}+2 p\right) g_{y p}+q^{2}\left(q^{2}+3 p\right) g_{z p} \\
& \quad-2 q g_{x q}-q^{2} g_{y q}-q\left(q^{2}+2 p\right) g_{z q}+q g_{y}+q^{2} g_{z}-4 g_{p}=0 \\
& 2 p^{2} g_{p p}-\left(q^{2}+2 p\right) g_{q q}+p q^{2} g_{x p}-2 p^{2} q g_{y p}-p^{2} q^{2} g_{z p} \\
& \quad+q\left(q^{2}+2 p\right) g_{x q}-p q^{2} g_{y q}+2 p^{2} q g_{z}+q^{2} g_{x}-p q g_{y}+4 p g_{p}=0 \\
& p g_{p p}-g_{p q}-g_{q q}+q^{2} g_{x p}-2 p q g_{y p}-p q^{2} g_{z p}+2 q g_{x q}+q^{2} g_{y q}+q\left(q^{2}+2 p\right) g_{z q} \\
& \quad-q^{2}\left(q^{2}+2 p\right) g_{x z}+p q^{3} g_{y z}-p^{2} q^{2} g_{z z}+2 g_{p}-q^{2} g_{x x}-q^{3} g_{x y}+p q^{2} g_{y y}-q g_{y}=0
\end{aligned}
$$

where $p=z_{x}$ and $q=z_{y}$.

It is possible to show that the general solution of the equations (2.4)-(2.7) is

$$
\begin{aligned}
h= & z_{y y}+c_{1}\left(z_{y}^{4}+\left(3 z_{x}+4 x\right) z_{y}^{2}+3 y z_{y}+\left(z_{x}+2 x\right)^{2}+2 z\right) \\
& +c_{2}\left(z_{y}^{3}+\left(2 z_{x}+3 x\right) z_{y}+2 y\right)+c_{3}\left(z_{y}^{2}+z_{x}+2 x\right)+c_{4} z_{y}+c_{5} .
\end{aligned}
$$

Hence the differential constraint $h=0$ is compatible with the Gibbons-Tsarev equation (2.1). In the case $c_{1}=c_{2}=c_{3}=0$ we obtain the differential constraint

$$
z_{y y}+c_{4} z_{y}+c_{5}=0 .
$$

From (2.8) we find the following representation

$$
z=a_{1} \exp \left(-c_{4} y\right)-c_{5} y / c_{4}+a_{2},
$$

where $a_{1}$ and $a_{2}$ depend on $x$. Substituting (2.9) into (2.1) we derive two ordinary differential equations

$$
a_{2}^{\prime \prime}+1=0, \quad a_{1}^{\prime \prime}+c_{5} a_{1}^{\prime}-c_{4}^{2} a_{1} a_{2}^{\prime}=0 .
$$

The first equation has the solution

$$
a_{2}=-x^{2} / 2+c_{6} x+c_{7}, \quad c_{6}, c_{7} \in R .
$$

In this case the second equation is

$$
a_{1}^{\prime \prime}+c_{5} a_{1}^{\prime}+c_{4}^{2}\left(x-c_{6}\right) a_{1}=0 .
$$


Setting $a_{1}=\exp \left(-c_{5} x / 2\right) v(x)$ we obtain the equation

$$
v^{\prime \prime}+(A+B x) v=0, \quad A, B \in R .
$$

According to [10] the solutions of the last equation can be expressed in terms of Airy functions.

It can be shown that the linear determining equation (2.2) has the following solution of third order

$$
\begin{aligned}
h= & z_{y y y}+c_{1}\left(3 z_{y}^{5}+\left(10 z_{x}+12 x\right) z_{y}^{3}+6 y z_{y}^{2}+\left(6 z_{x}^{2}+18 x z_{x}+2 z+12 x^{2}\right) z_{y}+4 y z_{x}+6 x y\right) \\
& +c_{2}\left(5 z_{y}^{4}+\left(12 z_{x}+15 x\right) z_{y}^{2}+6 y z_{y}+3 z_{x}^{2}+10 x z_{x}+15 / 2 x^{2}+z\right) \\
& +c_{3}\left(2 z_{y}^{3}+\left(3 z_{x}+4 x\right) z_{y}+y\right)+c_{4}\left(3 z_{y}^{2}+2 z_{x}+3 x\right)+c_{5} z_{y}+c_{6} .
\end{aligned}
$$

The corresponding constants $b_{1}$ and $b_{2}$ in (2.2) are given by

$$
b_{1}=2, \quad b_{2}=-2 .
$$

In the case $c_{1}=c_{2}=c_{3}=c_{6}=0$ and $c_{5}=-1$ the function $h$ gives the differential constraint

$$
z_{y y y}-z_{y}=0 .
$$

From (2.10) we obtain the following representation

$$
z=s_{1}(x)+s_{2}(x) e^{y}+s_{3}(x) e^{-y} .
$$

The functions $s_{1}(x), s_{2}(x)$ and $s_{3}$ must satisfy the equations

$$
s_{2}^{\prime \prime}-s_{1}^{\prime} s_{2}=0, \quad s_{1}^{\prime \prime}-2 s_{3} s_{2}^{\prime}-2 s_{2} s_{3}^{\prime}+1=0, \quad s_{3}^{\prime \prime}-s_{1}^{\prime} s_{3}=0 .
$$

If $s_{3}=a s_{2}$ then the last system reduces to the two equations

$$
s_{2}^{\prime \prime}-s_{1}^{\prime} s_{2}=0, \quad s_{1}^{\prime \prime}-4 a s_{2} s_{2}^{\prime}+1=0, \quad a \in R .
$$

Integrating the second equation, we find that

$$
s_{1}^{\prime}=-x-b+2 a s_{2}^{2}, \quad b \in R .
$$

We can insert this expression in (2.11) and obtain the second-order equation

$$
s_{2}^{\prime \prime}+\left(x+b-2 a s_{2}^{2}\right) s_{2}=0 .
$$

Using the transformations $t_{1}=x+b$ and $w=\sqrt{a} s_{2}$, we take the equation in $s_{2}$ to the second Painlevé equation [11]

$$
w^{\prime \prime}=2 w^{3}-t_{1} w
$$

The differential constraint

$$
z_{y y y}=0
$$

leads to a solution of (2.1) which is expressed in terms of elementary functions. 
3. Invariant solutions under involutive distributions. In this section we introduce invariant solutions under involutive distributions. Suppose that a collection of $p$ vector fields

$$
X_{s}=\sum_{i=1}^{n} \xi_{s}^{i}(x) \partial_{x_{i}}
$$

is given on an open set $U \subset R^{n}$. If this collection is linearly disconnected, i.e., the rank of the matrix $\left|\xi_{s}^{i}(x)\right|$ equals $p$ for all $x \in U$ and satisfies the involution condition

$$
\left[X_{i}, X_{j}\right]=\sum_{k=1}^{p} c_{i j}^{k}(x) X_{k}, \quad \forall 1 \leq i, j \leq p,
$$

where $c_{i j}^{k}$ are smooth functions, then this collection generates an involutive $p$-dimensional distribution $D_{p}$. A collection of vector fields with these properties is called an involutive basis or just a basis. It is well known that a distribution $D_{p}$ is involutive if and only if it possesses at least one involutive basis.

Definition. A solution $u=\varphi$ to a system of partial differential equation $E$ is invariant under an involutive distribution $D_{p}$ if $D_{p}$ is tangent to the manifold $S=$ $\{(x, u): u=\varphi(x)\}$. Obviously, the invariance of a solution under $D_{p}$ amounts to its invariance under the operators of an arbitrary involutive basis for $D_{p}$.

Now, consider the system of evolution equations

$$
u_{t}^{i}=F^{i}\left(t, x, u, u_{\alpha}\right), \quad i=1, \ldots, m
$$

where $t$ and $x=\left(x_{1}, \ldots, x_{n}\right)$ are independent variables, $u^{1}, \ldots, u^{m}$ are functions, $u=$ $\left(u^{1}, \ldots, u^{m}\right)$, and $u_{\alpha}$ stands for various partial derivatives with respect to $x_{1}, \ldots, x_{n}$. Denote the total derivatives with respect to $t$ and $x_{i}$ by the symbols $D_{t}$ and $D_{x_{i}}$.

Let $J^{k}\left(U, R^{m}\right)$ be the space of $k$-jets on $U \subset R^{n}$. Recall that a manifold $H \subset$ $J^{k}\left(R^{n+1}, R^{m}\right)$, defined by the equations

$$
h^{j}\left(t, x, u, u_{\beta}\right)=0, \quad j=1, \ldots, s,
$$

is an invariant manifold for (3.2) if the following identity holds on the set $[E] \cap[H]$ :

$$
D_{t} h^{j}=0
$$

Here $[E]$ and $[H]$ stand for the differential consequences of (3.2) and (3.3) with respect to $x_{1}, \ldots, x_{n}$. Denote the involutive distribution generated by vector fields $X_{1}, \ldots, X_{r}$ by $\left\langle X_{1}, \ldots, X_{r}\right\rangle$.

LEMMA 1. Suppose that vector fields

$$
X_{k}=\sum_{i=1}^{n} \xi_{k}^{i}(t, x, u) \partial_{x_{i}}+\sum_{j=1}^{m} \eta_{k}^{j}(t, x, u) \partial_{u^{j}}, \quad k=1, \ldots, n,
$$


generate an involutive distribution and that $\operatorname{det}\left(\xi_{k}^{i}\right) \neq 0$. If the manifold defined by the equations

$$
h_{k}^{j}=\sum_{i=1}^{n} \xi_{k}^{i} u_{x_{i}}^{j}-\eta_{k}^{j}=0, \quad 1 \leq j \leq m, 1 \leq k \leq n,
$$

is invariant with respect to (3.2) then system (3.2) has invariant solutions under this involutive distribution.

Proof. Write down the collection of fields $X_{1}, \ldots, X_{n}$ in vector form as follows:

$$
X=\xi \partial_{x}+\eta \partial_{u}
$$

Acting by the matrix $\xi^{-1}$ on $X$, we obtain the involutive collection

$$
Z=\partial_{x}+\tilde{\eta} \partial_{u}
$$

where $\tilde{\eta}=\xi^{-1} \eta$. The distribution $\left\langle Z_{1}, \ldots, Z_{n}\right\rangle$ is involutive.

The invariant solutions under $\left\langle X_{1}, \ldots, X_{n}\right\rangle$ must satisfy (3.5). The invariant solutions under $\left\langle Z_{1}, \ldots, Z_{n}\right\rangle$ must satisfy the equations

$$
u_{x_{k}}^{j}=\tilde{\eta}_{k}^{j}(t, x, u)
$$

Obviously, (3.5) and (3.6) have the same solutions. Since $Z$ is an involution distribution, the Poisson bracket $\left[Z_{i}, Z_{k}\right]$ vanishes. Consequently, we have

$$
Z_{i}\left(\tilde{\eta}_{k}^{j}\right)=Z_{k}\left(\tilde{\eta}_{i}^{j}\right)
$$

which means that the consistency conditions for (3.6) are satisfied.

Using (3.6) and inserting the derivatives of the functions $u^{j}$ with respect to $x_{k}$ in the right-hand side of (3.2), we come to the system

$$
u_{t}^{j}=G^{j}(t, x, u), \quad j=1, \ldots, m .
$$

By the Frobenius theorem, the system of (3.6) and (3.7) is compatible if the relations

$$
D_{x_{k}} G^{j}=D_{t} \tilde{\eta}_{k}^{j}, \quad j=1, \ldots, m ; k=1, \ldots, n
$$

are valid by virtue of (3.7) and (3.8). Validity of these conditions follows from the invariance of (3.5) with respect to (3.2). Indeed, this invariance means that

$$
D_{t}\left(u_{x_{k}}^{j}-\tilde{\eta}_{k}^{j}\right)=D_{x_{k}} F^{j}-D_{t} \tilde{\eta}_{k}^{j}=0 .
$$

Inserting the derivatives with respect to $x_{k}$ in (3.9), we see that (3.9) coincides with (3.8).

REMARK. If an involutive distribution is generated by analytic vector fields $X_{1}, \ldots, X_{p}$, where $p<n,(3.2)$ is a system of first-order equations with analytic righthand sides, and the rank of the matrix $\left(\xi_{k}^{i}\right)$ equals $p$, then (3.2) has an invariant solution relative to $X_{1}, \ldots, X_{p}$. The proof is carried out by the above scheme, but instead of the Frobenius theorem we should use the Riquier theorem on the existence of analytic solutions to an autonomous system with analytic right-hand sides [5]. 
To exemplify the application of a distribution to constructing solutions, consider the equation

$$
u_{t}=\Delta \ln u, \quad \Delta=\frac{\partial^{2}}{\partial x^{2}}+\frac{\partial^{2}}{\partial y^{2}},
$$

which arises in various application $[\mathbf{1 2}, \mathbf{1 3}]$ and possesses an infinite-dimensional algebra of point symmetries [14]. Some exact solutions to this equation can be found in $[15, \mathbf{1 6}]$. We give a solution to this equation which is invariant relative to the pair of commuting operators

$$
\begin{aligned}
& X_{1}=\partial_{x}-\left(u^{2}+\left(t u^{2}-x u^{2}+u\right) \tan (t)\right) \partial_{u}, \\
& X_{2}=\partial_{y}-\left(t u^{2}+u-x u^{2}\right) \partial_{u} .
\end{aligned}
$$

The corresponding manifold for these vector fields is

$$
\begin{aligned}
& u_{x}+u^{2}+\left(t u^{2}-x u^{2}+u\right) \tan (t)=0, \\
& u_{y}+t u^{2}+u-x u^{2}=0
\end{aligned}
$$

It is easy to verify that this is an invariant manifold for (3.10). Note that the vector fields $X_{1}$ and $X_{2}$ do not belong to the algebra of symmetries of (3.10). The general solution to (3.11), (3.12) and (3.10) has the form

$$
u=\frac{1}{A[\exp ((x-t) \tan (t)+y)] \cos t+x-t}, \quad A \in R .
$$

To use vector fields and distributions, we need a method for finding them. The classical approach to constructing vector fields relative to which the given differential equations are invariant was proposed by S. Lie. A modern exposition with many examples and new results was given by L. V. Ovsyannikov [6].

A determining equation enables us to find differential constraints compatible with the original equation. In the case of differential equations in more than two independent variables, we can propose systems of defining equations which would enable us to find involutive distributions.

Consider the system of involution equations (3.2) and the manifold in $J^{1}\left(U, R^{m}\right)$ defined by

$$
h_{j}^{i}=u_{x_{j}}^{i}+g_{j}^{i}(t, x, u)=0,
$$

where $i=1, \ldots, m$, and $j=1, \ldots, n$.

THEOREM. Suppose that the manifold (3.13) is invariant under the system (3.2) whose right-hand sides are polynomials in derivatives whose coefficients depend on $t, x_{1}, \ldots, x_{n}$ and $u^{1}, \ldots, u^{m}$. Then the functions $h_{j}^{i}$ satisfy the following system:

$$
D_{t} h_{j}^{i}+\left.m_{i j}(h)\right|_{[E]}=0, \quad 1 \leq i \leq m, 1 \leq j \leq n .
$$

Here $m_{i j}(h)$ is some operator representing a polynomial in $h_{l}^{k}, D_{x_{1}} h_{l}^{k}, \ldots, D_{x_{n}} h_{l}^{k}, \ldots, D^{\alpha} h_{l}^{k}$ $(k=1, \ldots, m, l=1, \ldots, n)$. The operators $m_{i j}(h)$ vanish whenever all $h_{l}^{k}$ are zero.

Proof. We first show that the total derivative of $h_{j}^{i}$ with respect to $t$ is representable as

$$
D_{t} h_{j}^{i}=m_{i j}(h)+\gamma_{i j}
$$


where $m_{i j}$ are operators whose shape is described in the theorem and $\gamma_{i j}$ are functions which may depend only on $t, x$ and $u$.

The following identities are valid on $[E]$ :

$$
D_{t} h_{j}^{i}=D_{x_{j}} F^{i}+\frac{\partial g_{j}^{i}}{\partial t}+\sum_{k=1}^{m} F^{k} \frac{\partial g_{j}^{i}}{\partial u^{k}} .
$$

Let $\frac{\partial^{|s|} u^{k}}{s^{s}}$ be a derivative of maximal order on the right-hand side of (3.16) and $s_{p} \neq 0$ ifor $s^{2}$ some $p$. By (3.16) and the assumptions of the theorem, this derivative enters (3.16) polynomially. Using (3.13), we can write down this derivative as follows:

$$
D_{x_{1}}^{s_{1}} \cdots D_{x_{p}}^{s_{p}-1} \cdots D_{x_{n}}^{s_{n}}\left(h_{p}^{k}\right)-D_{x_{1}}^{s_{1}} \cdots D_{x_{p}}^{s_{p}-1} \cdots D_{x_{n}}^{s_{n}}\left(g_{p}^{k}\right) .
$$

Note that the second summand involves no derivatives of order $|s|$ and is a polynomial in derivatives. Thus, all derivatives of maximal order on the right-hand side of (3.16) can be expressed in terms of the total derivatives of the functions $h_{q}^{r}(r=1, \ldots, m$, and $q=1, \ldots, n)$. Afterwards, it is possible to express the derivatives of order $|s|-1$, etc. down to the first-order derivatives.

We are left with demonstrating that the functions $\gamma_{i j}$ in (3.15) are all zero. By the conditions of the theorem, the manifold (3.13) is an invariant manifold for (3.2). Consequently, the following identity holds on $[E] \cap[H]$ :

$$
m_{i j}(h)+\gamma_{i j}=D_{t} h_{j}^{i}=0 .
$$

Since the $m_{i j}$ 's vanish on $[H]$, the functions $\gamma_{i j}$ are zero on $[E] \cap[H]$. Once the $m_{i j}$ 's are independent of the derivatives of the functions $u^{k}$, all $m_{i j}$ are identically zero.

REMARK. As we see from the proof of the theorem, the choice of the operators $m_{i j}$ is not uniquely defined.

For example, consider the second-order equation in three independent variables:

$$
u_{t}=G \equiv F^{1} u_{x x}+F^{2} u_{y y}+F^{3} u_{x}^{2}+F^{4} u_{y}^{2}+F^{5},
$$

where $F^{i}$ are some functions depending on $u$. Suppose that

$$
h_{1} \equiv u_{x}+g_{1}(t, x, y, u)=0, \quad h_{2} \equiv u_{y}+g_{2}(t, x, y, u)=0
$$

define an invariant manifold for (3.17). To derive a system of determining equations like (3.14), we express the derivatives $D_{t} h_{1}$ and $D_{t} h_{2}$ in terms of $h_{i}, D_{x} h_{i}, D_{y} h_{i}, D_{x}^{2} h_{i}, D_{x} D_{y} h_{i}$, and $D_{y}^{2} h_{i}(i=1,2)$. By (3.17), the following holds:

$$
D_{t} h_{1}=D_{x} G+\frac{\partial g_{1}}{\partial t}+\frac{\partial g_{1}}{\partial u} G .
$$

It is easy to verify that the right-hand side of the last equality is representable as

$$
\begin{gathered}
m_{11}\left(h_{1}, h_{2}\right)=G_{u_{x x}} D_{x}^{2} h_{1}+G_{u_{y y}} D_{y}^{2} h_{1}+\left[G_{u_{x}}+D_{x}\left(G_{u_{x x}}\right)\right] D_{x} h_{1}+G_{u_{y}} D_{y} h_{1} \\
+D_{x}\left(G_{u_{y y}}\right) D_{y} h_{2}+\left[G_{u}-D_{x}^{2}\left(G_{u_{x x}}\right)-D_{y}^{2}\left(G_{u_{y y}}\right)+r_{1}\right] h_{1}+s_{1} h_{2}+\gamma_{1}
\end{gathered}
$$

where $r_{1}, s_{1}$, and $\gamma_{1}$ are functions depending on $h_{1}, h_{2}$, and $G$. Since (3.18) is an invariant manifold, the function $\gamma_{1}$ equals 0 . Consequently, the first defining equation has the 
form

$$
D_{t} h_{1}=m_{11}\left(h_{1}, h_{2}\right)
$$

To obtain the second defining equation

$$
D_{t} h_{2}=m_{12}\left(h_{1}, h_{2}\right)
$$

we should replace $h_{1}$ in (3.12) with $h_{2}, x$ with $y, r_{1}$ with $r_{2}$, and $s_{1}$ with $s_{2}$. The following lemma asserts that, under some conditions, solutions to equations like (3.13) enable us to construct differential constraints compatible with the system of evolution equations (3.2). It is worth noting that the form of the operators $m_{i j}$ is unimportant, provided that only $m_{i j}(0)=0$.

LEMMA 2. Suppose that the functions

$$
h_{j}^{i}=\sum_{s=1}^{n} \xi_{j}^{s}(t, x, u) u_{x_{s}}^{i}-g_{j}^{i}(t, x, u)
$$

satisfy a system like (3.14) on $[E]$ with $m_{i j}(0)=0$. If the vector fields

$$
X_{j}=\sum_{s=1}^{n} \xi_{j}^{s} \partial_{x_{s}}+\sum_{i=1}^{m} g_{j}^{i} \partial_{u_{i}}, \quad j=1, \ldots, n
$$

generate an involutive distribution and $\operatorname{det}\left(\xi_{j}^{s}\right) \neq 0$ then there is a solution to the system of (3.2) and the equations

$$
h_{j}^{i}=0, \quad i=1, \ldots, m, \quad j=1, \ldots, n .
$$

Proof. Since the functions $h_{j}^{i}$ satisfy (3.14), in view of $m_{i j}(0)=0$ (3.20) defines an invariant manifold for (3.2). To complete the proof, it suffices to refer to Lemma 1.

Finding solutions to general nonlinear equations (3.14) might represent a very complicated problem. To simplify the problem, we remove all terms nonlinear in $h_{l}^{k}$ from the operators $m_{i j}$ as was done above in the case of an evolution equation with one space variable. In result, we obtain some linear equation

$$
D_{t} h_{j}^{i}+l_{i j}(h)=0 \text {. }
$$

As we have done above, multiply the coefficients of the operators $l_{i j}$ by undetermined constants and write down the resultant equations as

$$
D_{t} h_{j}^{i}+L_{i j}(h)=0
$$

calling them linear determining equations (LDEs). For example, the LDEs for (3.17) have the form

$$
\begin{gathered}
D_{t} h_{1}=L_{11}\left(h_{1}, h_{2}\right) \equiv a_{1} G_{u_{x x}} D_{x}^{2} h_{1}+a_{2} G_{u_{y y}} D_{y}^{2} h_{1}+\left[a_{3} G_{u_{x}}+a_{4} D_{x}\left(G_{u_{x x}}\right)\right] D_{x} h_{1}+a_{5} G_{u_{y}} D_{y} h_{1} \\
+a_{6} D_{x}\left(G_{u_{y y}}\right) D_{y} h_{2}+\left[a_{7} G_{u}+a_{8} D_{x}^{2}\left(G_{u_{x x}}\right)+a_{9} D_{y}^{2}\left(G_{u_{y y}}\right)\right] h_{1}, \\
D_{t} h_{2}=L_{12}\left(h_{1}, h_{2}\right),
\end{gathered}
$$


where $L_{12}\left(h_{1}, h_{2}\right)$ is obtained from $L_{11}\left(h_{1}, h_{2}\right)$ by replacing $h_{1}$ with $h_{2}, x$ with $y$, and $a_{i}$ with $b_{i}$.

Although the above arguments were for systems of evolution equations, we can try to extend them to a more general situation. Assume given a system

$$
n_{i}(u)=F^{i}\left(t, x, u, u_{\alpha}\right), \quad i=1, \ldots, m,
$$

where $n_{i}$ are linear differential operators with constant coefficients and the right-hand sides are similar to those in the evolution systems (3.2). To find the functions $h_{j}^{i}$, we suggest using the following equation in place of (3.21):

$$
N_{i}\left(h_{j}^{i}\right)+L_{i j}(h)=0,
$$

where the operators $N_{i}$ are obtained from $n_{i}$ by replacing partial derivatives with total derivatives. Alongside (3.23), it is useful to introduce the following analog of B-defining equations [17]:

$$
N_{i}\left(h_{j}^{i}\right)+L_{i j}(h)+\sum_{\substack{1 \leq l \leq m \\ 1 \leq k \leq n}} b_{l j}^{k i} h_{k}^{l}=0,
$$

where $1 \leq i \leq m, 1 \leq j \leq n$, and $b_{l j}^{k i}$ are functions that may depend on $t, x$, and $u$.

We call equations of the form (3.23) quasilinear determining equations (QDEs). We exhibit an example of QDEs in finding involutive distributions. Consider one of the nonlinear dispersion models describing the propagation of long two-dimensional waves [22]:

$$
\eta_{t t}=g d \Delta \eta+\frac{d^{2}}{3} \Delta \eta_{t t}+\frac{3}{2} g \Delta \eta^{2}
$$

where $\eta(t, x, y)$ is the deviation of a fluid from an equilibrium state, $d$ is the depth of the unperturbed fluid, and $g$ is the free fall acceleration. By translations and dilations, we can reduce this equation to the form

$$
u_{t t}-\Delta\left(u_{t t}\right)-u \Delta u-(\nabla u)^{2}=0 .
$$

In accordance with the above method, the QLEs for (3.25) have the form

$$
\begin{aligned}
& D_{t}^{2} h_{1}-D_{t}^{2} D_{x}^{2} h_{1}-D_{t}^{2} D_{y}^{2} h_{1}+a_{1} u\left(D_{x}^{2} h_{1}+D_{y}^{2} h_{1}\right)+a_{2} u_{x} D_{x} h_{1}+a_{3} u_{y} D_{y} h_{1} \\
& \quad+a_{4} u_{x} D_{y} h_{2}+\left(a_{5} \Delta u+a_{6} u_{x x}+a_{7} u_{y y}+r_{1}\right) h_{1}+q_{1} h_{2}=0 \\
& \quad D_{t}^{2} h_{2}-D_{t}^{2} D_{x}^{2} h_{2}-D_{t}^{2} D_{y}^{2} h_{2}+b_{1} u\left(D_{x}^{2} h_{2}+D_{y}^{2} h_{2}\right)+b_{2} u_{y} D_{y} h_{2}+b_{3} u_{x} D_{x} h_{2} \\
& \quad+b_{4} u_{y} D_{x} h_{1}+\left(b_{5} \Delta u+b_{6} u_{x x}+b_{7} u_{y y}+r_{2}\right) h_{2}+q_{2} h_{1}=0
\end{aligned}
$$

where $a_{i}$ and $b_{i}$ are constants, and $r_{j}$ and $q_{j}$ are functions which may depend on $t, x, y$, and $u$ and which should be found together with $h_{1}$ and $h_{2}$. The scheme for solving (3.26) and (3.27) is completely analogous to the standard scheme of group analysis of differential equations $[\mathbf{6}, \mathbf{1 8}]$. For this reason, we omit all intermediate computations and set forth only the final results.

If $h_{1}$ and $h_{2}$ are sought in the form corresponding to the point symmetries

$$
\begin{aligned}
& h_{1}=\xi_{1}^{1} u_{t}+\xi_{2}^{1} u_{x}+\xi_{3}^{1} u_{y}+\eta^{1}, \\
& h_{2}=\xi_{1}^{2} u_{t}+\xi_{2}^{2} u_{x}+\xi_{3}^{2} u_{y}+\eta^{2},
\end{aligned}
$$


where $\xi^{i}$ and $\eta^{j}$ are functions of $t, x, y$, and $u$, then under the condition $\left(\xi_{1}^{1}\right)^{2}+$ $\left(\xi_{3}^{1}\right)^{2}+\left(\xi_{1}^{2}\right)^{2}+\left(\xi_{2}^{2}\right)^{2} \neq 0$ equations (3.26) and (3.27) can be shown to have solutions leading only to admissible operators for (3.25). There appear new solutions only when

$$
h_{1}=u_{x}+g_{1}(t, x, y, u), \quad h_{2}=u_{y}+g_{2}(t, x, y, u)
$$

The final form of $g_{1}$ and $g_{2}$ is as follows:

$$
g_{1}=s_{1} x+s_{2} y+s_{3}, \quad g_{2}=s_{2} x+s_{4} y+s_{5}
$$

Moreover, the functions $s_{i}(i=1, \ldots, 5)$ depend only on $t$ and satisfy the following system of five second-order differential equations:

$$
\begin{aligned}
& s_{1}^{\prime \prime}+3 s_{1}^{2}+s_{1} s_{4}+2 s_{2}^{2}=0, \\
& s_{2}^{\prime \prime}+3 s_{1} s_{2}+3 s_{2} s_{4}=0, \\
& s_{3}^{\prime \prime}+3 s_{1} s_{3}+2 s_{2} s_{5}+s_{3} s_{4}=0, \\
& s_{4}^{\prime \prime}+s_{1} s_{4}+2 s_{2}^{2}+3 s_{4}^{2}=0, \\
& s_{5}^{\prime \prime}+s_{1} s_{5}+2 s_{2} s_{3}+3 s_{4} s_{5}=0 .
\end{aligned}
$$

For completeness of exposition, we write down the constants $a_{i}$ and $b_{i}(i=1, \ldots, 7)$ and the functions $r_{j}$ and $q_{j}(j=1,2)$ in (3.26) and (3.27) corresponding to $g_{1}$ and $g_{2}$ :

$$
\begin{aligned}
& a_{1}=b_{1}=a_{4}=b_{4}=-1, \quad a_{2}=b_{2}=a_{3}=b_{3}=-3, \\
& a_{5}=a_{6}=a_{7}=b_{5}=b_{6}=b_{7}=0, \\
& r_{1}=3 s_{1}+s_{4}, r_{2}=s_{1}+3 s_{4}, \quad q_{1}=2 s_{1}, \quad q_{2}=2 s_{2} .
\end{aligned}
$$

The functions $h_{1}$ and $h_{2}$ generate the differential constraints

$$
\begin{aligned}
& u_{x}+s_{1} x+s_{2} y+s_{3}=0, \\
& u_{y}+s_{2} x+s_{4} y+s_{5}=0 .
\end{aligned}
$$

These constraints enable us to find the following representation for a solution to (3.25):

$$
u=\frac{-s_{1} x^{2}}{2}-s_{2} x y-\frac{s_{4} y^{2}}{2}-s_{3} x-s_{5} y+s_{6} .
$$

Inserting this in (3.25), we obtain the following equation for $s_{6}$ :

$$
s_{6}^{\prime \prime}=3 s_{1}^{2}+2 s_{1} s_{4}-s_{1} s_{6}+4 s_{2}^{2}+s_{3}^{2}+3 s_{4}^{2}-s_{4} s_{6}+s_{5}^{2} .
$$

The system of the six differential equations in the six functions $s_{i}$ deserves further study. For example, it would be interesting to find a solution expressible via elementary functions.

Acknowledgement. Work supported by Russian Foundation for Basic Research (No. 04-01-00130). 


\section{REFERENCES}

1. E. Goursat, Leçons sur l'intégration des équations aux dérivées partielles du second ordre a deux variables indépendantes. T. II. (Paris: Librairie scientifique A. Hermann, 1898).

2. A. F. Sidorov, V. P. Shapeev and N. N. Yanenko, Method of Differential Constraints and its Applications to Gasdynamics (Nauka, Novosibirsk, 1984) (in Russian).

3. G. Darboux Sur la théorie des équations aux dérivées partielles. Comptes Rendus. Vol. $\operatorname{LXX}(1870), 746-749$.

4. A. R. Forsyth, Theory of differential equations. Part IV. Partial differential equations. Vol. VI (Cambridge University Press, 1906).

5. J. E. Pommaret, Systems of Partial Differential Equations and Lie Pseudogroups (Gordon $\&$ Breach, 1978).

6. L. V. Ovsyannikov, Group Analysis of Differential Equations (Academic Press, 1982).

7. O. V. Kaptsov, Linear determining equations for differential constraints, Sbornik: Mathematics 189 (1998), 1839-1854.

8. O. V. Kaptsov and I. V. Verevkin, Differential constraints and exact solutions of nonlinear diffusion equations. J. Phys. A: Math. Gen. 36 (2003), 1401-1414.

9. J. Gibbons and S. P. Tsarev, Conformal maps and reductions of the Benney equations, Phys. Lett. A 258 (1999), No. 4-6, 263-271.

10. F. W. J. Olver, Asymptotics and Special Functions (Academic Press, 1974).

11. E. L. Ince, Ordinary differential equations (Dover, New York, 1956).

12. S. N. Aristov, Periodic and localized exact solutions to the equation $h_{t}=\Delta \log h$. Prikl. Mekh. Tekhn. Fiz. 40 (1999), No. 1, 22-26.

13. V. V. Pukhnachov, Multidimensional exact solutions of a nonlinear diffusion equation, Prikl. Mekh. Tekhn. Fiz. 36 (1995), No. 2, 23-31.

14. G. A. Nariboli, Self-similar solutions of some nonlinear equations, Appl. Sci. Res. 22 (1970), 449-461.

15. V. A. Dorodnitsyn, I. V. Knyazeva and S. R. Svirshchevski, Group properties of the heat equation with a source in two-dimensional and three-dimensional cases. Differentsial'nye Uravneniya 19 (1983), No. 7, 1215-1223.

16. V. A. Galaktionov, Invariant subspaces and new explicit solutions to evolution equations with quadratic nonlinearities, Proc. Roy. Soc. Edinburgh Sect. A 125 (1995), No. 2, 225-246.

17. V. K. Andreev, O. V. Kaptsov, V. V. Pukhnachev and A. A. Rodionov, Applications of Group-Theoretic Methods in Hydrodynamics (Kluwer Academic Publishers, 1998).

18. P. J. Olver, Applications of Lie groups to differential equations (Springer-Verlag, 1986). 